\title{
CHANNEL ESTIMATION USING TYPE-III EVEN DISCRETE COSINE TRANSFORM IN MULTICARRIER COMMUNICATIONS
}

Elena Domínguez-Jiménez, David Luengo

Universidad Politécnica de Madrid (Spain)
Fernando Cruz-Roldán

Universidad de Alcalá (Spain)

\begin{abstract}
In this work, we address the problem of channel estimation in multicarrier communications by means of the Type-III even discrete cosine transform (DCT3e). We present a novel efficient procedure based on using the DCT3e both at the transmitter and the receiver. The proposed approach does not require adding any redundancy or knowing the exact length of the channel's impulse response. It suffices to use any symmetric training sequence at the transmitter with enough leading and tail zeros. We demonstrate that an accurate estimation of the channel's impulse response can be attained, and illustrate the good behavior of the proposed scheme using ITU-T pedestrian channel $\mathrm{B}$.
\end{abstract}

Index Terms - MCM, Channel estimation, DCT.

\section{INTRODUCTION}

Multicarrier modulation (MCM) has become the preferred technique in current state-of-the-art digital communication systems like mobile communications (3GPP LTE), wireless local/metropolitan area networks (WiFi/WiMax), digital subscriber lines (DSL), digital TV broadcasting (DVB-T) or power line communications (PLC). Most of these systems are based on the discrete Fourier transform (DFT), and the resulting MCM schemes are usually referred to collectively as orthogonal frequency division multiplexing (OFDM) [1, 2]. These systems require the estimation of the channel's impulse response, which is usually unknown and time-varying. To estimate the channel, training symbols (known both by the transmitter and the receiver) are often used [3]. However, in the last years discrete cosine transforms (DCTs) have been considered as an alternative to the DFT for MCM systems, due to their improved robustness with respect to some of the main weaknesses of OFDM systems. For instance, several authors have established the better performance of DCT-based MCM schemes under carrier frequency offset [4-8] for signal reconstruction at the receiver.

This work has been partially supported by the Ministerio de Economía, Industria y Competitividad (Spain) through projects TEC2015-64835-C3-3R and TEC2015-64835-C3-1. E. Domínguez-Jiménez (ORCiD code: 00000003-1016-6963) and D. Luengo (ORCiD code: 0000-0001-7407-3630) are members of UPM's TACA Research Group and would like to thank UPM for its support. The first author is affiliated to ETSI Industriales (UPM).
Regarding the channel estimation problem, recent works have presented effective procedures for several DCTs: the discrete cosine transform Type-I even (DCT1e) has been studied in [9, 11], whereas [10] analyzes both the Type-II even DCT (DCT2e) and the Type-IV even DCT (DCT4e). In order to complete the study for the different classes of DCTs, it remains to explore the use of the DCT Type-III even (DCT3e) for channel estimation. In this work, we present a novel procedure to estimate any channel filter in MCM systems by using the DCT3e. The key advantages of the DCT3e that we exploit in this work are the following:

- The convolution-multiplication property of two vectors is fulfilled in the DCT3e domain, if one of the two vectors presents whole-point symmetry (WS) and contains enough leading and tail zeros $[12,13]$.

- Signals which present zero coefficients at even positions are transformed into vectors with whole-point symmetry (WS) in the DCT3e transform domain.

The first property enables the estimation of non-symmetric channels without introducing any additional transform in the receiver by constructing a symmetric training signal with enough leading and tail zeros. The second property can be exploited when considering sparse training symbols.

The work is organized as follows. First, in Section 2 we recall the general MCM scheme and the resulting channel estimation problem. Then, the proposed channel estimation procedure based on the DCT3e is presented in Section 3, and its behaviour is illustrated in Section 4 through simulations. Finally, some conclusions are drawn in Section 5.

\section{PROBLEM STATEMENT: CHANNEL ESTIMATION IN MULTICARRIER MODULATION}

Fig. 1 shows the general block diagram representing the transceiver and the channel estimation stage herein considered [10]. At the transmitter, the incoming data $\mathbf{X}$ are processed by an $N$-point inverse transform $\mathbf{T}_{a}^{-1}$, with $N$ being the number of subchannels or subcarriers. Then, matrix $\Gamma$ introduces redundancy in order to ensure that the linear convolution performed by the channel corresponds to an element-by-element operation in the transformed domain. 


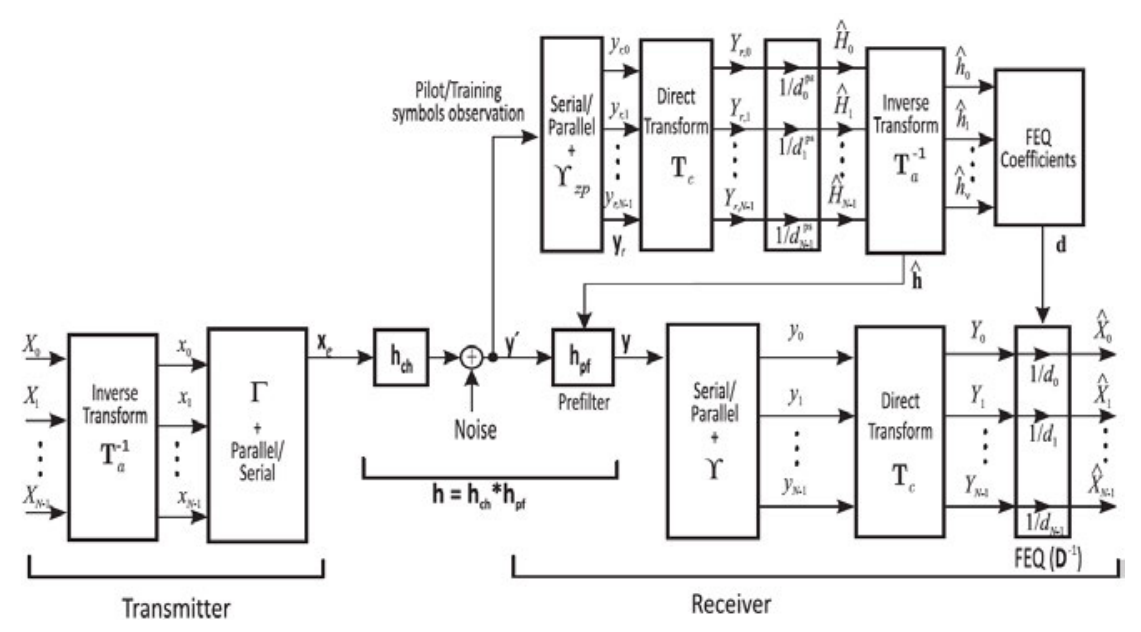

Fig. 1. General block diagram for channel estimation in a multicarrier modulation (MCM) system.

For example, in OFDM the underlying transform is the DFT, and $\boldsymbol{\Gamma}$ introduces the required cyclic prefix/suffix; whereas for the DCT approach, the corresponding redundancy matrices $\boldsymbol{\Gamma}$ are given in [6]. In particular, when using the DCT3e, $\boldsymbol{\Gamma}$ produces a whole-point symmetry (WS) on the left and a whole-point antisymmetry (WA) on the right of the original signal. At the receiver, we observe two stages in Fig 1:

1. The channel estimation stage, which is carried out from time to time in order to achieve an estimation $\widehat{\mathbf{h}}_{c h}$ of the time-varying channel's impulse response $\mathbf{h}_{c h}$. This stage is developed by using a pilot/training symbol, known a priori. The filter is estimated by means of a -possibly different- transform $\mathbf{T}_{c}$ and the 1-tap coefficients $\left(d_{0}^{p s}, \ldots, d_{N-1}^{p s}\right)$, which depend on the pilot symbol. The obtained $\widehat{\mathbf{h}}_{c h}$ is then used to compute a prefilter in the following stage, $\mathbf{h}_{p f}$, that ensures the required symmetry when non-symmetric signals are transmitted.

2. The signal reconstruction stage, which is carried out when the channel filter is known (or has been estimated by the channel estimation stage). It recovers the original time-domain data symbol vector $\mathrm{x}$ transmitted through the channel, by means of another set of 1-tap equalizers $\left(d_{0}, \ldots, d_{N-1}\right)$ which depend on the channel's filter, already known at this stage.

In this work, we focus on the channel estimation stage using the DCT3e transform; with this approach, no other transforms are needed, i.e., $\mathbf{T}_{a}=\mathbf{T}_{c}=\mathbf{C}_{3 e}$, defined matricially as [12]:

$$
\left[\mathbf{C}_{3 e}\right]_{k, j}=2 a_{j} \cos \left(\frac{(2 k+1) j \pi}{2 N}\right), 0 \leq k, j \leq N-1,
$$

where

$$
a_{j}= \begin{cases}\frac{1}{2}, & \text { if } j=0 \\ 1, & \text { otherwise }\end{cases}
$$

\section{CHANNEL ESTIMATION FOR DCT3E-BASED MULTICARRIER MODULATION}

We now focus on the channel estimation problem of Fig. 1, by using the DCT3e both at the transmitter and the receiver. Without loss of generality, we assume that the maximum length $L$ of the channel filter $\mathbf{h}$ is odd (otherwise, we simply introduce an extra null component), so $L=2 \nu+1<N$, with $N=2 M+2 L-1$, and we apply the following procedure:

1. Build a training symbol with WS symmetry, whose first and last $\nu=(L-1) / 2$ components are null:

$$
\mathbf{x}=[\underbrace{0, \ldots, 0}_{\nu}, \underbrace{x_{M}, \ldots, x_{1}, x_{0}, x_{1}, \ldots, x_{M}}_{2 M+1}, \underbrace{0, \ldots, 0}_{\nu}]^{T} .
$$

2. After transmitting $\mathbf{x}$ through the channel, the received data, $\mathbf{y}=\mathbf{x} * \mathbf{h}+\mathbf{n}$, has length $N=2 M+2 L-1$.

3. Following the approach of [10], we express the convolution matricially as $\mathbf{y}=\widetilde{\mathbf{X}} \cdot \mathbf{h}+\mathbf{n}$, where $\widetilde{\mathbf{X}}$ is a lower triangular $N \times L$ Toeplitz matrix:

$$
\widetilde{\mathbf{X}}=\left[\begin{array}{rrrr}
\multicolumn{3}{c}{\mathbf{O}_{\nu \times L}} & \\
x_{M} & 0 & \ddots & 0 \\
\vdots & x_{M} & \ddots & \vdots \\
x_{1} & \ddots & \ddots & 0 \\
x_{0} & \ddots & \ddots & x_{M} \\
\vdots & \ddots & \ddots & \vdots \\
x_{M} & \ddots & \ddots & x_{0} \\
0 & x_{M} & \ddots & x_{1} \\
\vdots & \ddots & \ddots & \vdots \\
0 & \cdots & 0 & x_{M}
\end{array}\right]
$$


4. By denoting as $\mathbf{h}_{z p}^{T}=\left[\begin{array}{lll}\mathbf{0}_{M+\nu} & \mathbf{h}^{T} & \mathbf{0}_{M+\nu}\end{array}\right]$ the zeropadded channel's impulse response, let us notice that the convolution can also be written as

$$
\mathbf{x} * \mathbf{h}=\widetilde{\mathbf{X}} \cdot \mathbf{h}=\mathbf{X}_{\text {equiv }} \cdot \mathbf{h}_{z p}
$$

where $\mathbf{X}_{\text {equiv }}$ is an $N \times N$ square matrix, that can be written as $\mathbf{X}_{\text {equiv }}=\mathbf{X}_{T}+\mathbf{X}_{H}$, being $\mathbf{X}_{T}$ a Toeplitz matrix and $\mathbf{X}_{H}$ a Hankel-type matrix:

$$
\begin{aligned}
& \mathbf{X}_{T}=\left[\begin{array}{rrrrrrr}
x_{0} & x_{1} & \cdots & x_{M} & 0 & \cdots & 0 \\
x_{1} & \ddots & \ddots & & & \ddots & \vdots \\
\vdots & \ddots & \ddots & \ddots & & & 0 \\
x_{M} & & \ddots & \ddots & & & x_{M} \\
0 & & & & & & \vdots \\
\vdots & \ddots & & & & & \\
0 & \cdots & 0 & x_{M} & \cdots & x_{1} & x_{0}
\end{array}\right], \\
& \mathbf{X}_{H}=\left[\begin{array}{rrrrrrr}
0 & x_{1} & \cdots & x_{M} & \cdots & 0 & 0 \\
\vdots & \vdots & . \cdot & 0 & . \cdot & \vdots & 0 \\
0 & x_{M} & . \cdot & & . \cdot & 0 & -x_{M} \\
0 & 0 & . \cdot & & . \cdot & -x_{M} & \vdots \\
\vdots & \vdots & . \cdot & & . \cdot & \vdots & \vdots \\
0 & 0 & \ldots & -x_{M} & \cdots & -x_{3} & -x_{2}
\end{array}\right]
\end{aligned}
$$

In this way, the result given in [13, p. 2635] guarantees that $\mathbf{X}_{\text {equiv }}$ is diagonalized by the DCT3e:

$$
\mathbf{C}_{3 e} \cdot \mathbf{X}_{\text {equiv }} \cdot \mathbf{C}_{3 e}^{-1}=\mathbf{D} \text {. }
$$

Besides, the diagonal entries of matrix $\mathbf{D}$ are themselves the DCT3e of the $N$-length right-half vector $\mathbf{x}_{z p}^{r}=\left[x_{0}, \ldots, x_{M}, 0, \ldots, 0\right]^{T}[13]$ :

$$
[\mathbf{D}]_{k, k}=d_{k}^{p s}=\left[\mathbf{C}_{3 e} \cdot \mathbf{x}_{z p}^{r}\right]_{k} .
$$

Notice that these coefficients can be pre-computed and stored in memory for the training signal of choice.

5. We apply the DCT3e at the receiver, and denote as $\mathbf{Y}=$ $\mathbf{C}_{3 e} \cdot \mathbf{y}$ the received vector in the transformed domain, $\mathbf{H}=\mathbf{C}_{3 e} \cdot \mathbf{h}_{z p}$ the transformed vector associated to the zero padded channel's impulse response, and $\mathbf{N}=$ $\mathbf{C}_{3 e} \cdot \mathbf{n}$, the transformed noise vector. Hence, we get the expression in the transformed domain

$$
\mathbf{Y}=\mathbf{D} \cdot \mathbf{H}+\mathbf{N}
$$

Finally, we obtain an estimation of $\mathbf{H}$ as

$$
\widehat{\mathbf{H}}_{k}=\mathbf{Y}_{k} / d_{k}^{p s}, \quad k=0, \ldots, N-1,
$$

and compute

$$
\mathbf{C}_{3 e}^{-1} \cdot \widehat{\mathbf{H}}=\widehat{\mathbf{h}}
$$

which provides a perfect estimation of $\left[\mathbf{0}_{M+\nu} \mathbf{h} \mathbf{0}_{M+\nu}\right]$ in the absence of noise.
Thus, we have been able to find a simple and efficient solution to the channel estimation problem for an MCM system based on the DCT3e, with no need of extra transforms.

\subsection{Our proposed training symbol}

Now we present a training signal which is optimal for the proposed approach. It is constructed in the DCT3e domain as follows:

$$
X_{k}=2 \cos \left(\frac{(2 k+1)(N-1) \pi}{4 N}\right), 0 \leq k \leq N-1 .
$$

If we choose this signal, after applying the $\mathbf{C}_{3 e}^{-1}$ block at the transmitter, we obtain $\mathbf{C}_{3 e}^{-1} \cdot \mathbf{X}$, which only has a non null component. Furthermore, using this training symbol the diagonal entries $d_{k}^{p s}$ are the optimal ones:

$$
d_{k}^{p s}=\left[\mathbf{C}_{3 e} \cdot \mathbf{x}_{z p}^{r}\right]_{k}=1,
$$

and we can ensure that

$$
\widehat{\mathbf{H}}=\mathbf{Y}=\mathbf{C}_{3 e} \cdot \mathbf{y}, \quad k=0, \ldots, N-1 .
$$

Hence, no extra computation is needed at the receiver and the estimated channel is simply $\widehat{\mathbf{h}}=\mathbf{y}$.

\section{NUMERICAL RESULTS}

In this section, we analyze the behaviour of the proposed channel estimation scheme by testing it on one of the standardized ITU-R M.1225 channels [14]. First of all, a training signal is constructed in the DCT3e domain by setting $\mathbf{X}=\mathbf{C}_{3 e} \cdot[0, \ldots, 0,1,0, \ldots, 0]^{T}$. The inverse DCT3e of $\mathbf{X}$ is performed, the first and last $\nu=(L-1) / 2$ zeros (out of the $L-1$ leading and tail zeros) are removed, and the resulting length $N-(L-1)$ time-domain signal, $x_{m}$, is transmitted. ${ }^{1}$ After passing this signal through the channel, with impulse response $h_{m}$ for $0 \leq m \leq L-1$, we obtain an $N$-length time-domain signal, $z_{m}=[\mathbf{x} * \mathbf{h}]_{m}$. Then, zero-mean additive white Gaussian noise (AWGN) with variance $\sigma_{n}^{2}$ is added, resulting in a received signal

$$
y_{m}=[\mathbf{x} * \mathbf{h}]_{m}+n_{m}=\sum_{r=0}^{N-1} h_{r} x_{m-r}+n_{m}
$$

where $n_{m} \sim \mathcal{N}\left(0, \sigma_{n}^{2}\right)$ is the AWGN, with $\mathcal{N}\left(\mu, \sigma^{2}\right)$ denoting a univariate Gaussian density with mean $\mu$ and variance $\sigma^{2}$. The $N$-length DCT3e of $y_{m}\left(Y_{k}\right)$ is then computed and used to estimate the DCT3e of the channel's impulse response $\left(\widehat{H}_{k}\right)$ as described in Section 3. Finally, the $N$-length inverse DCT3e of $\widehat{H}_{k}$ is obtained and the relevant central samples are extracted to obtain the estimate of the channel's impulse response, $\hat{h}_{m}$.

\footnotetext{
${ }^{1}$ Note that all this process can be avoided simply by pre-computing $\mathbf{x}$ and storing it.
} 


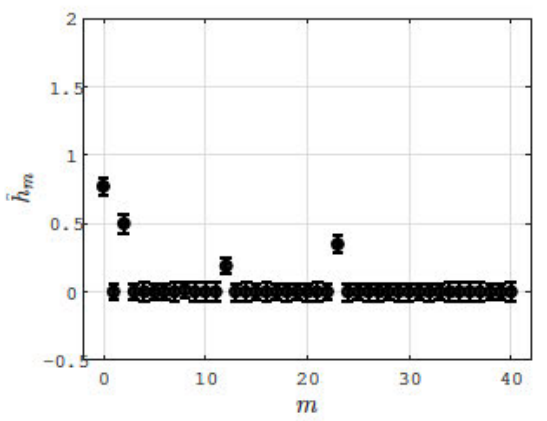

(a)

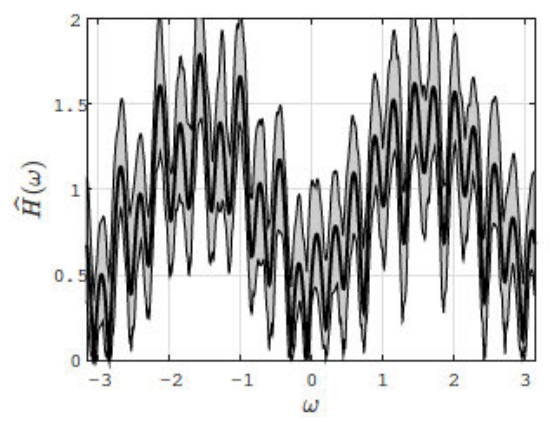

(d)

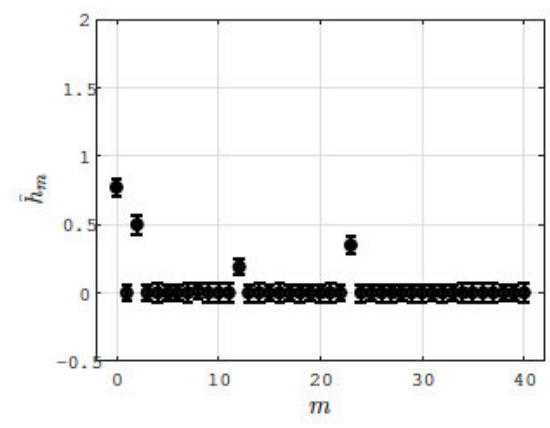

(b)

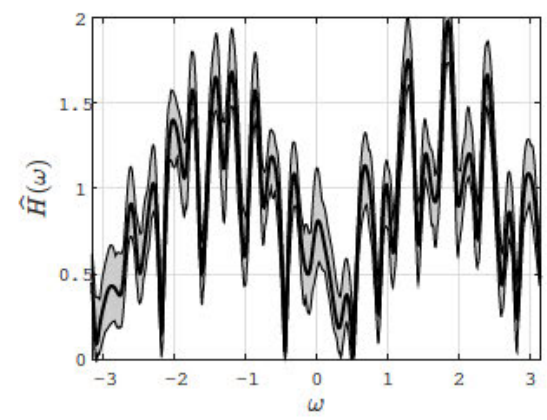

(e)

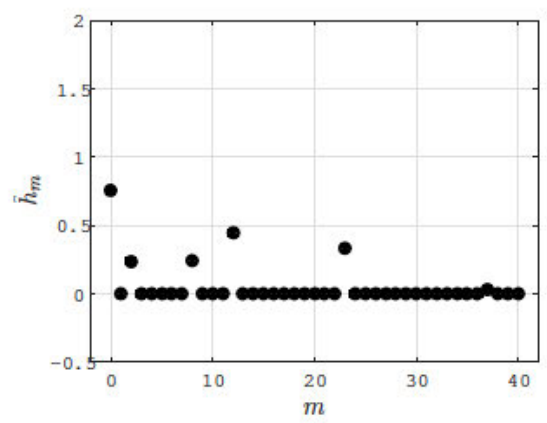

(c)

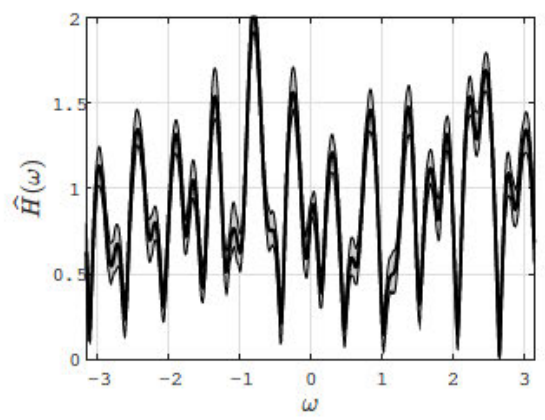

(f)

Fig. 2. (a)-(c): Estimated channel's impulse response (true value and two times the standard deviation) for SNR $=0 \mathrm{~dB}$ (a), SNR $=5 \mathrm{~dB}(\mathrm{~b})$, and $\mathrm{SNR}=10 \mathrm{~dB}$ (c). (d)-(f): Estimated channel's frequency response (true value and range between maximum and minimum estimated values) for $\mathrm{SNR}=0 \mathrm{~dB}$ (d), SNR $=5 \mathrm{~dB}$ (e), and SNR $=10 \mathrm{~dB}$ (f). In all cases, $N=1023$.

As mentioned above, we consider one of the channels standardized by ITU-R for the evaluation of radio transmission technologies for IMT 2000 [14]. More precisely, we address the estimation of the ITU-T M.1225 pedestrian channel B for $N=1023$. The channels were generated using Matlab's stdchan function using a carrier frequency $f_{c}=2 \mathrm{GHz}$, a maximum Doppler shift $f_{d} \approx 9.266 \mathrm{~Hz}$, and a sampling period $T_{s}=100 \mathrm{~ns}$. With this sampling period, the channel's impulse response becomes $h_{m}=A_{0} \delta_{m}+$ $A_{2} \delta_{m-2}+A_{8} \delta_{m-8}+A_{12} \delta_{m-12}+A_{23} \delta_{m-23}+A_{37} \delta_{m-37}$, where $\delta$ denotes Kronecker's delta and each of the $A_{i}$ are complex-valued, independent random variables with Rayleigh distributed amplitudes and uniform phases. Note that the length of the channel's impulse response is actually $L^{\prime}=38$, but we set $L=41$ for the simulations in order to show the robustness of the proposed approach.

Fig. 2 shows three examples of the reconstructed channel (both in the time and frequency domains) for $N=1023$ and different SNRs. Note that the channel's reconstruction is already very good for SNR $=0 \mathrm{~dB}$ (Figs. 2(a) and 2(b)) and it becomes almost perfect for SNR $=10 \mathrm{~dB}$ (Figs. 2(c) and 2(d)). In the time domain, the average values of the estimated coefficients of the channel's impulse response are always equal to the true values (i.e., there is no bias) and the standard deviation is small. In the frequency domain, all the peaks and valleys of the estimated channel's frequency response are located in the same positions as those of the true channel's frequency response.

\section{CONCLUSIONS}

In this work, we have presented a general procedure for the estimation of an arbitrary channel's impulse response when the Type-III even Discrete Cosine Transform (DCT3e) is used within a multicarrier modulation system. The proposed approach is based on the construction of a symmetric training signal with enough leading and tail zeros. The scheme proposed here requires using only the DCT3e both in the transmitter and the receiver: no other transform is necessary, because the channel is estimated using only the DCT3e, thus leading to a very efficient hardware implementation. Furthermore, no assumptions are required about the channel, except for knowing the maximum possible length of its impulse response. Numerical simulations show that the proposed algorithm is able to provide very accurate channel reconstruction in noisy environments for the ITU-T M. 1225 pedestrian channel B. Future research lines include developing DCT-based semi-blind channel estimation schemes. 


\section{REFERENCES}

[1] R. V. Nee and R. Prasad, "OFDM for wireless multimedia communications," Artech House, 2000.

[2] A. R. Bahai, B. R. Saltzberg and M. Ergen, "Multicarrier digital communications: theory and applications of OFDM,' Springer, 2004.

[3] M. Morelli and U. Mengali, "A comparison of pilotaided channel estimation methods for OFDM systems," IEEE Transactions on signal processing 49 (12): 30653073, Dec. 2001.

[4] N. Al-Dhahir, "Optimum DCT-Based Multicarrier Transceivers for Frequency-Selective Channels", IEEE Trans. Communications54 (5): 911-921, May 2006.

[5] P. Tan and N.C. Beaulieu, "A comparison of DCT-Based OFDM and DFT-Based OFDM in Frequency Offset and Fading Channels", IEEE Trans. Communications 54 (11): 2113-2125, Nov. 2006.

[6] F. Cruz-Roldán, M. E. Domínguez-Jiménez, G. Sansigre-Vidal, P. Amo-López, M. Blanco-Velasco, and Á. Bravo-Santos, "On the Use of Discrete Cosine Transforms for Multicarrier Communications," IEEE Trans. Signal Processing 60 (11): 6085-6090, Nov. 2012.

[7] P. Kumar and P. Kumar, "Performance evaluation of modified OFDM for underwater communications", 2013 IEEE International Conference on Communications Workshops (ICC), pp. 967-971, 9-13 June 2013.

[8] F. Cruz-Roldán, M. E. Domínguez-Jiménez, G. Sansigre-Vidal, J. Piñeiro-Ave and M. Blanco-Velasco, "Single-Carrier and Multicarrier Transceivers based on Discrete Cosine Transform Type-IV,' IEEE Trans. Wireless Communications 12 (12): 6454-6463, Dec. 2013.

[9] M. E. Domínguez-Jiménez, D. Luengo and G. SansigreVidal, "Estimation of Symmetric Channels for Discrete Cosine Transform Type-I Multicarrier Systems: A Compressed Sensing Approach," The Scientific World Journal, pp. 1-11, 2015.

[10] F. Cruz-Roldán, M. E. Domínguez-Jiménez, G. Sansigre-Vidal, D. Luengo and M. Moonen, "DCTbased channel estimation for single-and multicarrier communications," Signal Processing 128: 332-339, 2016.

[11] M. E. Domínguez-Jiménez, D. Luengo, G. SansigreVidal and F. Cruz-Roldán, "A Novel Channel Estimation Scheme for Multicarrier Communications with the Type-I even Discrete Cosine Transform ," Proceedings of the 25th European Signal Processing Conference (EUSIPCO 2017), pp. 2303-2307, Sept. 2017.
[12] S. A. Martucci, "Symmetric Convolution and the Discrete Sine and Cosine Transforms, " IEEE Trans. Signal Processing 42 (5): 1038-1051, May 1994.

[13] V. Sánchez, P. García, A. M. Peinado, J. C. Segura, and A. J. Rubio, "Diagonalizing properties of the Discrete Cosine Transforms" IEEE Trans. Signal Processing 43 (11): 2631-2641, Nov. 1995.

[14] Recommendation ITU-R M.1225, “Guidelines for Evaluation of Radio Transmission Technologies for IMT2000," 1997. 